Kompass

Autoimmun

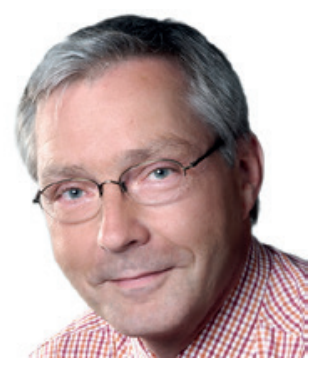

\title{
Die Wahl des Antidiabetikums bei bullösen Pemphigoiden entsprechend anpassen
}

\author{
Michael Sticherling \\ Hautklinik, Universitätsklinikum Erlangen, Deutsches Zentrum für Immuntherapie, Erlangen, Deutschland
}

Abstract aus Silverii GA, Dicembrini I, Nreu B, et al.: Bullous pemphigoid and dipeptidyl peptidase-4 inhibitors: a meta-analysis of randomized controlled trials. Endocrine 2020;69:504-507.

\section{Keywords}

DPP-4 inhibitors · Meta-analysis · Bullous pemphigoid

\begin{abstract}
Purpose: An increasing body of evidence suggests that dipeptidyl-peptidase 4 (DPP-4) inhibitors could play a role in the development of bullous pemphigoid. The knowledge regarding this association is based on case reports, pharmacovigilance database analyses, and observational studies. Data from randomized clinical trials are a relevant source of information on adverse events. Since no single trial has a sufficient power to assess the risk of very rare adverse events, such as pemphigoid, metanalyses of RCTs could be a useful tool for exploring this issue.
\end{abstract}

Methods: An extensive Medline, Embase and Cochrane Database search for sitagliptin or vildagliptin, omarigliptin or saxagliptin or alogliptin or trelagliptin or anagliptin or linagliptin or gemigliptin or evogliptin or teneligliptin was performed up to September 30th, 2019. All trials performed on type 2 diabetes, with duration $\geq 24$ weeks, and comparing DPP4i with placebo or active drugs were collected. The study has been registered on PROSPERO (\#153344). Mantel-Haenszel odds ratio (MH-OR) with 95\% Confidence Interval $(95 \% \mathrm{Cl})$ was calculated for pemphigoid. Results: A total of 138 eligible trials were identified (61,514 patients in DPP-4 inhibitors and 59,661 patients in the control group). Only six trials reported at least one case of pemphigoid (17 and 1 cases in DPP4i and control groups, respectively). DPP-4 inhibitors were associated with an increased risk of pemphigoid ( $\mathrm{MH}-\mathrm{OR}$ $4.44[1.31,15.00], p=0.020)$. A separate analysis for trials with linagliptin showed a significant increase of BP with the active drug (MH-OR 4.69 [1.09, 20.22]; $p=0.04$ ).

Conclusion: In conclusion, available data from randomized controlled trials seem to confirm the association between DPP-4 inhibitors and bullous pemphigoid. This association could be limited to one molecule of the class (i.e., linagliptin), although data on other DPP4-i (e.g., vildagliptin) are insufficient to rule out similar detrimental effects.

(c) 2020 Springer Science+Business Media, LLC, part of Springer Nature 


\section{Transfer in die Praxis}

\section{Hintergrund}

Das bullöse Pemphigoid (BP) ist in Westeuropa und den USA die häufigste autoimmunbullöse Dermatose, die mit steigender Inzidenz betont im höheren Lebensalter auftritt. Angesichts des zunehmenden Durchschnittsalters der Bevölkerung ist daher auch mit einer weiteren Zunahme seiner Häufigkeit zu rechnen. Die Medikamentenassoziation des BP ist bis heute umstritten. In der Hauptgruppe der Betroffenen im Alter von 70 Jahren besteht häufig eine umfangreiche Begleitmedikation. Antidiabetika sind in dieser Liste neben Antihypertonika führend, und in den letzten Jahren wurden hier die Dipeptidylpeptidase-4-Inhibitoren (DPP4i) am intensivsten untersucht.

\section{Zusammenhang DPP4i und Pemphigoid-Erkrankungen}

Silverii et al. präsentieren in ihrem Artikel eine Meta-Analyse von randomisierten kontrollierten Studien zu DPP4i oder Gliptinen, in deren Rahmen ein BP aufgetreten war. Recherchiert wurden dafür in MEDLINE, Embase und der Cochrane Database die verschiedenen Gliptine in klinischen Studien zum Typ-II-Diabetes mit mindestens 24 Wochen Dauer, die DPP4i mit Placebo oder anderen aktiven Wirkstoffen verglichen haben.

In insgesamt 138 Studien mit 61514 Patienten unter DPP4i und 69661 Patienten als Kontrollgruppe wurde die Mantel-Haenszel (MH)-Odds Ratio (OR) mit 95\%-Vertrauensintervall für einen Zusammenhang zum BP kalkuliert. Nur in 6 dieser Studien wurde mindestens 1 Fall von Pemphigoid beschrieben: insgesamt 17 Fälle in der Behandlungs- und 1 Patient in der Kontrollgruppe. Die OR der Gesamtgruppe der Gliptine für das Pemphigoid beträgt 4,44 mit einer statistischen Signifikanz von $p=0,020$, für Linagliptin beträgt sie 4,69, für Saxagliptin 4,96 und für Sitagliptin 3,0.

Insgesamt sind in dieser Meta-Analyse nur wenige Fälle beschrieben, wobei das BP in keiner Studie als klinischer Endpunkt definiert war. Auch erhielten die Patienten multiple andere Wirkstoffe, von denen einige mit dem BP assoziiert sind, wie zum Beispiel Furosemid. Da in den Publikationen meist keine vollständigen Listen von Nebenwirkungen enthalten sind, ist das BP in der Auswertung möglicherweise unterrepräsentiert. Auch sind das klinische Bild sowie die immunserologischen Daten der betroffenen Patienten nicht aufgeführt. Zusätzlich ist unklar, inwieweit bei Auftreten eines klinisch verdächtigen (und bekanntermaßen heterogenen) Bildes differenzialdiagnostisch überhaupt an ein BP gedacht und eine entsprechende Diagnostik eingeleitet worden ist.

\section{Bullöses Pemphigoid}

Klinisch finden sich neben einem deutlichen generalisierten Pruritus pralle Blasen auf erythematösem Grund mit einer Schleimhautbeteiligung bei 20-30\% der Betroffenen. Als Hauptzielantigen des BP wird das BP180/Kollagen XVII angesehen, das in der epidermalen Basalmembran lokalisiert ist und dessen Störung klinisch zu prallen, subepidermalen Blasen führt. Die entsprechenden Autoantikörper lassen sich in spezifischen ELISAs (Enzyme-linked Immuno- sorbent Assays) nachweisen und zur Diagnostik wie zum Verfolgen des Therapieverlaufes nutzen. Angesichts großer Heterogenität unter klinischen, therapeutischen und immunserologischen Aspekten handelt es sich um eine Gruppe verwandter Erkrankungen, die mittlerweile als Pemphigoid-Gruppe bezeichnet werden und unter denen das klassische BP am häufigsten ist. Die Auslöser des $\mathrm{BP}$ sind heterogen und bis heute nicht klar definiert, neben Umweltfaktoren und Infektionen werden Medikamente verdächtigt, die Proteinantigene der Basalmembran alterieren und immunologisch erkennbar machen oder die Immunabwehr beeinflussen, körpereigene Proteine als fremd zu erkennen und zu attackieren. Klinische Situationen, in denen ein Auslöser - nicht nur beim BP verdächtig ist, sind 1) das erstmalige Auftreten oder 2) erneute Ausbrechen, 3) die Verschlechterung einer vorbestehenden Erkrankung bei Einführung einer neuen Medikation oder Kontakt zu einem Umweltfaktor sowie 4) das Nichtansprechen auf gut wirksame Therapeutika. Nach Absetzen des verdächtigten Agens sollte die Erkrankung zurückgehen, konsequent wäre die erneute Exposition nach Abklingen der Beschwerden zur Überprüfung eines erneuten Ausbruches der Erkrankung, was jedoch im Einzelfall ethisch kritisch zu sehen ist.

\section{Dipeptidylpeptidase-4-Inhibitoren}

Dipeptidylpeptidase 4 (DPP-4) spaltet Dipeptide von Proteinen ab, wenn sich an zweiter Stelle der Aminosäuresequenz Prolin oder Arginin befinden. Durch diese katalytische Hydrolyse wird die biologische Wirkung von verschiedenen Proteinen entweder verstärkt, vermindert oder aufgehoben. Zu den durch DPP-4 gespaltenen Peptiden gehören Substanz P, Mellitin und Bradykinin, aber auch Glucagon-like Peptide 1 (GLP-1), das die Freisetzung von Insulin anregt. Eine Blockierung von DPP-4 vermindert den Abbau von GLP-1 und fördert damit indirekt die Insulinfreisetzung, vermindert aber gleichzeitig die Bildung von Glukagon. Pharmakologische Inhibitoren der DPP-4 sind als sogenannte Gliptine seit Oktober 2006 in den USA, mittlerweile aber auch in Europa zur Behandlung des Diabetes mellitus Typ II zugelassen. Ein bekanntes Mitglied ist das Sitagliptin. DDP-4-Blockierung zeigt zusätzlich eine fördernde Wirkung auf die Wundheilung sowie eine Reduktion der Immunantwort bei AIDS.

\section{Zusammenhang DPP4i und Pemphigoid-Erkrankungen}

Das Risiko der Entwicklung eines BP scheint nach allen vorliegenden Daten unter DPP4i tatsächlich erhöht zu sein. Die hier zitierte Arbeit zeigt eine deutlich erhöhte OR für Linagliptin und Saxagliptin, aber auch Sitagliptin. Ob es sich um einen Gruppeneffekt handelt oder nur einzelne Gliptine bedeutsam sind, ist noch unklar. Die katalytische Wirkung von DPP-4 verändert möglicherweise Proteinantigene der Basalmembranzone und führt bei entsprechend prädisponierten Menschen durch Induktion von spezifischen Autoantikörpern zur klinischen Manifestation eines BP. Auch in weiteren retrospektiven Untersuchungen sowie mit Pharmakovigilanz-Daten
66

Kompass Autoimmun 2021;3:65-67 DOI: $10.1159 / 000516089$ 
konnte der Zusammenhang gezeigt werden. Klinisch und serologisch haben andere Arbeiten eher widersprüchliche Daten publiziert, einerseits mit Betroffenen mit einem eher geringer inflammatorischen klinischen Bild, andererseits bei deutlicher männlicher Dominanz mit einer schwereren Krankheitsausprägung sowie einer prominenten Einbeziehung von Stamm und Kopf. So brauchten Patienten mit Linagliptin-assoziiertem BP demnach höhere Dosen von systemischen Kortikosteroiden bis zur Krankheitskontrolle als Patienten mit Vildagliptin-assoziiertem BP. Das Ab- bzw. Umsetzen des Antidiabetikums scheint daher die Therapie der Wahl zu sein und führt zu meist folgenlosem Abklingen der Erkrankung.

\section{Fazit für die Praxis}

Die DPP4i sind aktuell der Hit in der Liste verdächtigter medikamentöser Auslöser des BP. Die Ergebnisse sind zum Teil aber noch hete- rogen und widersprüchlich, obwohl die Fülle der Daten mittlerweile einen Zusammenhang nahelegt. Die genannten biologischen Wirkungen von DPP-4 sind möglicherweise für die Beteiligung an der BP-Pathogenese bedeutsam. Bisher sind jedoch nur phänomenologische Assoziationen zwischen BP und DPP4i belegt. Angesichts der aktuellen Daten sollten bei einem BP-Verdacht keine Gliptine eingenommen bzw. diese frühzeitig abgesetzt werden.

\section{Disclosure Statement}

Es besteht kein Interessenkonflikt zu diesem Thema.

Korrespondenz an:

Prof. Dr. Michael Sticherling, michael.sticherling@uk-erlangen.de 